\title{
A Self-Linking Field Formalism
}

\author{
Edwin Eugene Klingman \\ Cybernetic Micro Systems, Inc., San Gregorio, CA, USA \\ Email:klingman@geneman.com
}

How to cite this paper: Klingman, E.E. (2021) A Self-Linking Field Formalism. Journal of Modern Physics, 12, 440-452. https://doi.org/10.4236/jmp.2021.124031

Received: February 16, 2021

Accepted: March 12, 2021

Published: March 15, 2021

Copyright $\odot 2021$ by author(s) and Scientific Research Publishing Inc. This work is licensed under the Creative Commons Attribution International License (CC BY 4.0).

http://creativecommons.org/licenses/by/4.0/

\begin{abstract}
The Gauss-linking integral for disjoint oriented smooth closed curves is derived linking integrals from the Biot-Savart description of the magnetic field. DeTurck and Gluck extend this linking from 3-space $R^{3}$ to $S U(2)$ space of the unit 3-sphere and hyperbolic space in Minkowski $R^{1,3}$. I herein extend Gauss-linking to self-linking and develop the concept of self-dual, which is then applied to gravitomagnetic dynamics. My purpose is to redefine Wheeler's geon from unstable field structures based on the electromagnetic field to self-stabilized gravitomagnetic field structures.
\end{abstract}

\section{Keywords}

Gauss-Linking, Self-Linking, Biot-Savart Operator, Green's Function, Laplacian, Maxwell's Eqns, Gravitomagnetism, Self-Dual, Geons

\section{Introduction}

In several recent papers [1] [2] I have developed the theory of a self-interacting primordial field. Formulated in Hestenes' Geometric Calculus, the theory derives Heaviside's equations in a strength-independent manner. This differs from the usual derivation of the equations via linearization of Einstein's general relativistic field equations in that linearization is characterized as the weak field approximation. The implication of the new derivation is that not only weak fields but all gravitational fields obey Heaviside's equations. The fact that this appears to be true has been discussed by Clifford Will [3] and others, but no explanation has been offered.

My analysis of the Kasner metric for the primordial field [4] associates the metric with the gravitomagnetic field and emphasizes Vishwakarma's major point [5] that only density-based solutions exist. This, combined with all-strength solutions extends the theory of gravitation into physical ranges that have not been treated. The goal is to explore gravity in these previously ignored realms, based 
on, where feasible, analogy with Maxwell's field equations, upon which Heaviside modeled his theory of gravity.

The close resemblance between Maxwell's electromagnetic field equations and Heaviside's gravitomagnetic field theory and the fact that Einstein's non-linear field equations are compatible with Heaviside's equations, suggests that we explore the physics of gravity based on similarity of the field equations while also analyzing the differences between the fields. Probably the key difference is that electromagnetic fields, being uncharged, do not interact with themselves, while the gravitomagnetic field does interact with itself. Thus the electromagnetic field cannot source itself while the momentum of the gravitomagnetic field can source a derived field, and effectively link to itself. The question explored in this paper is whether this linkage supports stable structure. This is pursued by analyzing the linking of the field to its source, and, in particular, Gauss-linking formalism developed for the electromagnetic field by DeTurck, Gluck, and others.

Gauss-linking is claimed to have originated in computing the linking number of the earth's orbit with the orbits of certain asteroids. DeTurck and Gluck [6] derive Gauss-linking integrals from the Biot-Savart description of the magnetic field, then extend the linking from 3-space $\mathfrak{R}^{3}$ to the $S U(2)$-space of the unit 3 -sphere and hyperbolic space in Minkowski's $\mathfrak{R}^{1,3}$. This potentially extends Gauss-linking to Stern-Gerlach analysis based on $S U(2)$-space and equivalent geometric algebra of bivectors. In [7] I derive Hamiltonian physics based in Euclidean space in which Pythagorean distance is defined by $\mathrm{ds} s^{2}=\mathrm{d} t^{2}+\mathrm{d} x^{2} \rightarrow \infty$ while Minkowski invariant distance is defined by photon physics:

$\mathrm{d} s^{2}=\mathrm{d} t^{2}-\mathrm{d} x^{2}=0$, where photon speed $c=1$. This paper extends linking associated with the electromagnetic field to self-linking of the gravitomagnetic field. I ask whether self-stabilized field structures are possible in this context.

The goal of this paper is to extend the physical concepts and mathematical equations associated with Gauss-linking to self-linking in hope of deriving a framework capable of supporting calculation of self-stabilized field structures. The mathematics (calculus, topology and geometry) is complex; therefore we review the background in detail before extending Gauss- to self-linking.

The plan of this paper is as follows:

Section 1 introduces aspects of a new derivation of the law of gravity from a primordial field. The key aspect is that Heaviside's equations are strength independent. Also key is that solutions to the field equations in the Kasner metric are density-dependent. These suggest the possibility of stable field structures which we investigate in terms of Gauss-linking.

Section 2 introduces key terms and concepts associated with linking integrals in $\mathfrak{R}^{3}$. For completeness we define relevant concepts for $S^{3}$ and $H^{3}$; our focus will be on linking in three-space. The $S U(2)$-space relates primarily to spin and Minkowski invariance to inertial mass.

Section 3 introduces Geometric Calculus multi-vectors in field equations and multi-vector operators, including inverse operators. Green's function is then in- 
troduced and explained and the linking integral of section 1 is reformulated in terms of Green's function. This is shown to allow extension of Gauss-linking from $\mathfrak{R}^{3}$ to $S U(2)$ and Minkowski invariant formalisms.

Section 4 reviews derivation of field equations from discrete inverse operators and association of the Gauss-linking terms with current-based fields in the Biot-Savart framework.

Section 5 explores the Biot-Savart formula and the definition of helicity. These link Gauss-linked closed curves to electromagnetism and set up Calugareanu's specialization of Gauss-linking to twisting, writhing, and helicity, all of which are relevant to self-linked solitonic structures.

Section 6 introduces the concept of Link Duality, which is believed to be a novel result of this paper. The physical implications are presented for electromagnetic theory.

Section 7 introduces the concept of Helical Duality in terms of solenoidal aspects of electromagnetism. This concept is also believed to be a novel result of this paper.

Section 8 extends the concepts of the previous sections and formulates the symmetry and asymmetry associated with the linkage of charge to electromagnetic field.

Section 9 introduces the concept of self-dual field and discusses why electromagnetism fails to be self-dual. Gravito-magnetism is then explored in these terms and we observe that the gravitomagnetic field is dualistically self-linked. The key diagram of this paper is then shown and explained as the basis of the planned study of self-stabilized structures.

Section 10 summarizes the above; the conjectured self-dual formalism has been successfully developed, and will be explored in future in terms of self-stabilized structures.

\section{Relevant Background}

Consider two disjoint oriented smooth closed curves in Euclidean 3-space $\mathfrak{R}^{3}$, parameterized as $\kappa_{1}=\{\boldsymbol{x}(s)\}$ and $\kappa_{2}=\{\boldsymbol{y}(t)\}$ with $\alpha(\boldsymbol{x}, \boldsymbol{y})$ defined as the distance from $\boldsymbol{x}$ to $\boldsymbol{y}$. The linking number of these two curves is defined to be the intersection number of either one of them with an oriented surface bounded by the other. The symmetry is such that the linking number does not depend on which of the curves is used to bound the surface, nor on the type of surface chosen. The formalism is based on the Euclidean inner product in $\mathfrak{R}^{4}$, $(\boldsymbol{x}, \boldsymbol{y})=x_{0} y_{0}+x_{1} y_{1}+x_{2} y_{2}+x_{3} y_{3}$. For completeness we define relevant concepts for unit sphere $S^{3}=\left\{x \in R^{4}:(x, x)=1\right\}$ and Minkowski hyperboloid $H^{3}=\left\{\boldsymbol{x} \in \mathfrak{R}^{1,3}:(\boldsymbol{x}, \boldsymbol{x})=1\right.$ and $\left.x_{0}>1\right\}$ although our focus will be on linking in 3 -space. For example, the Biot-Savart law links circulation at a field point in 3-space, a distance $\boldsymbol{r}$ from a source point in 3-space. The topology, shown in Figure 1, shows two interlinked curves, $\kappa_{1}=\{\boldsymbol{x}(s)\}$ and $\kappa_{2}=\{\boldsymbol{y}(t)\}$, both of which are parameterized (by $s$ and $t$ respectively), with specific points $x(s)$ 


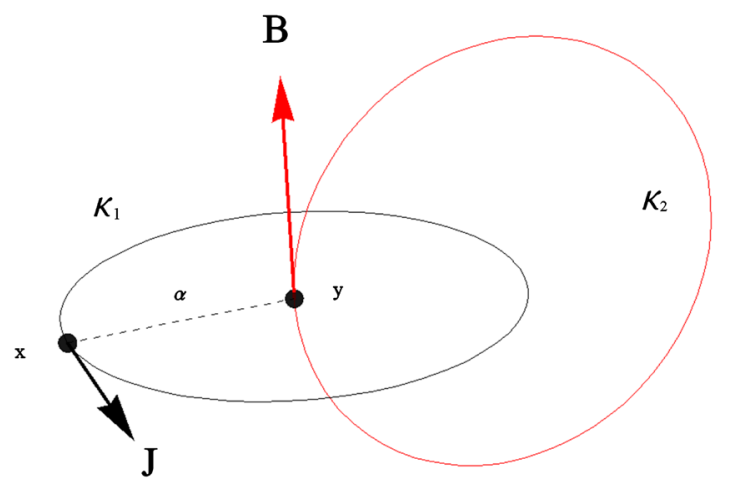

Figure 1. Two linked curves representative of the Gauss-linking problem.

and $\boldsymbol{y}(t)$ a distance $\alpha(x, y)=\boldsymbol{x}-\boldsymbol{y}$ apart. Also shown are the tangents at $\boldsymbol{x}(s)$ and $\boldsymbol{y}(t)$, denoted $\mathrm{d} \boldsymbol{x} / \mathrm{d} s$ and $\mathrm{d} y / \mathrm{d} t$ respectively. In these terms the Gauss-integral formula for linking numbers in Euclidian 3-space is given:

$$
\operatorname{Link}\left(\kappa_{1}, \kappa_{2}\right)=\frac{1}{4 \pi} \int_{\kappa_{1} \times \kappa_{2}} \frac{\mathrm{d} \boldsymbol{x}}{\mathrm{ds}} \times \frac{\mathrm{d} \boldsymbol{y}}{\mathrm{d} t} \cdot \frac{\boldsymbol{x}-\boldsymbol{y}}{|\boldsymbol{x}-\boldsymbol{y}|^{3}} \mathrm{~d} s \mathrm{~d} t
$$

It can be seen that if both tangents are in a plane, then $\frac{\mathrm{d} x}{\mathrm{~d} s} \times \frac{\mathrm{d} y}{\mathrm{~d} t}=0$ and there is no Gauss-linking.

\section{Field Equations and Inverse Field Equations}

We begin with Maxwell's equation for electrostatic field $\nabla \cdot \boldsymbol{E}=\rho\left(\varepsilon_{0}=1\right)$ and the definition of the field as gradient of potential $\boldsymbol{E}=-\nabla \phi$ where $\rho(x)$ is charge density distribution and potential $\phi \sim 1 / r$. The geometric algebra form of Maxwell's equations:

$$
\bar{\nabla} \bar{F}(x, t)=\bar{J}(x, t)
$$

where $\bar{\nabla}=\left(\nabla+\frac{1}{c} \frac{\partial}{\partial t}\right)$ is the multi-vector differential operator, $\bar{F}(x, t)$ is the multi-vector field and $\bar{J}(x, t)$ is a multi-vector source and the overbar indicates multi-vector. The general form (including wave equations) is

$$
\hat{L} \bar{F}(x)=\bar{J}(x)
$$

where $\hat{L}$ is an operator formed from a linear combination of linear operators: $1, \nabla, \nabla^{2}, \partial_{t}, \partial_{t t}$. The solutions have a linear structure and the most general solution has the form

$$
\bar{F}(x, t)=\int \mathrm{d}^{3} x^{\prime} \mathrm{d} t^{\prime} G\left(x, x^{\prime} ; t, t^{\prime}\right) \bar{J}\left(x^{\prime}, t^{\prime}\right)+\bar{F}_{0}(x, t)
$$

where $F_{0}$ is any solution of $L F_{0}=0$ and $G\left(x, x^{\prime} ; t, t^{\prime}\right)$ is the Green's function to be determined. To understand the meaning of the Green's function rewrite Equations (3) and (4) to obtain

$$
\bar{J}(x, t)=\hat{L} \bar{F}(x, t)=\hat{L}\left(\int \mathrm{d}^{3} x^{\prime} \mathrm{d} t^{\prime} G\left(\boldsymbol{x}, \boldsymbol{x}^{\prime} ; t, t^{\prime}\right) \bar{J}\left(\boldsymbol{x}^{\prime}, t^{\prime}\right)\right)
$$

Since the integral is over $x^{\prime}$ and $t^{\prime}$ and the linear operators are with respect 
to $x$ and $t$ we can move the operators inside the integral to obtain

$$
\bar{J}(\boldsymbol{x}, t)=\int \mathrm{d}^{3} x^{\prime} \mathrm{d} t^{\prime} \hat{L} G\left(\boldsymbol{x}, \boldsymbol{x}^{\prime} ; t, t^{\prime}\right) \bar{J}\left(\boldsymbol{x}^{\prime}, t^{\prime}\right)
$$

We observe that the source at point $\boldsymbol{x}$ is given as an integral over all values $x^{\prime}$ in the region. If this integral is to reduce to $J(x, t)$ then function $L G\left(\boldsymbol{x}, \boldsymbol{x}^{\prime} ; t, t^{\prime}\right)$ must pick out the value $\boldsymbol{x}$ from all possible values $\boldsymbol{x}^{\prime}$ hence the generalized Green's function must behave like a Dirac delta

$$
\hat{L} G\left(x, x^{\prime} ; t, t^{\prime}\right)=\delta\left(x-x^{\prime}\right) \delta\left(t-t^{\prime}\right)
$$

For static fields a Green's function for the Laplacian $\Delta=\nabla \cdot \nabla$ is

$G\left(\boldsymbol{x}, \boldsymbol{x}^{\prime}\right)=\frac{-1}{4 \pi\left\|\boldsymbol{x}-\boldsymbol{x}^{\prime}\right\|}$ and the Laplacian-based delta function in $\mathfrak{R}^{3}$ is $\delta\left(\boldsymbol{x}-\boldsymbol{x}^{\prime}\right)=\frac{-1}{4 \pi} \nabla^{2}\left(\frac{1}{\left\|\boldsymbol{x}-\boldsymbol{x}^{\prime}\right\|}\right)$. If we define $\varphi(\alpha)=1 / \alpha$ and substitute it into linking Equation (1) we obtain the equivalent:

$$
\operatorname{Link}\left(\kappa_{1}, \kappa_{2}\right)=\frac{1}{4 \pi} \int_{\kappa_{1} \times \kappa_{2}} \frac{\mathrm{d} \boldsymbol{x}}{\mathrm{d} s} \times \frac{\mathrm{d} \boldsymbol{y}}{\mathrm{d} t} \cdot \nabla_{y} \varphi(x, y) \mathrm{d} s \mathrm{~d} t
$$

where differentiation $\nabla_{y}$ is with respect to the $y$-variable and function $\varphi$ is the fundamental solution of the Laplacian $\Delta \varphi=\delta$. In extending the linking integral from $\mathfrak{R}^{3}$ to $S^{3}$ and $H^{3}$, DeTurck and Gluck retain the Equation (7) integral but redefine the solution of the Laplacian, $\varphi_{0}$

$$
\begin{array}{cc}
\mathfrak{R}^{3}: \varphi_{0}(\alpha)=-\left(\frac{1}{4 \pi \alpha}\right) & \Delta \varphi=\delta \\
S^{3}: \varphi_{0}(\alpha)=-\left(\frac{1}{4 \pi \alpha}\right)(\pi-\alpha) \csc (\alpha) & \Delta \varphi-\varphi=\delta \\
H^{3}: \varphi_{0}(\alpha)=-\left(\frac{1}{4 \pi \alpha}\right) \operatorname{csch}(\alpha) & \Delta \varphi+\varphi=\delta
\end{array}
$$

In short, field equations of the form $\Delta \varphi=s$ have a solution based on the inverse anti-derivative:

$$
\Delta \varphi=s \Rightarrow \varphi=\left(\Delta^{-1}\right) s, \Delta^{-1}=\int_{M} G+\oint_{\partial M} G
$$

where the Green's function is calculated over the region inside the boundary and optionally a second integral of the derivatives normal to the bounding surface of the field. Boundaries are often chosen primarily to simplify the field solution. We know from vector calculus $\nabla\left(\frac{1}{r}\right)=\frac{r}{r^{3}}$ and we know that $\varphi(r)=-\frac{1}{4 \pi r}$ is the fundamental solution of the Laplacian in $\mathfrak{R}^{3}: \Delta \varphi=\delta$ where $\delta$ is the Dirac delta function. Thus Gauss-linking of Equation (1) is equivalent to Equation (7):

$$
\begin{aligned}
& \operatorname{Link}\left(\kappa_{1}, \kappa_{2}\right)=\frac{1}{4 \pi} \int_{\kappa_{1} \times \kappa_{2}} \frac{\mathrm{d} \boldsymbol{x}}{\mathrm{d} s} \times \frac{\mathrm{d} \boldsymbol{y}}{\mathrm{d} t} \cdot \frac{\boldsymbol{x}-\boldsymbol{y}}{|\boldsymbol{x}-\boldsymbol{y}|^{3}} \mathrm{~d} s \mathrm{~d} t \\
& \Rightarrow \frac{1}{4 \pi} \int_{\kappa_{1} \times \kappa_{2}} \frac{\mathrm{d} \boldsymbol{x}}{\mathrm{d} s} \times \frac{\mathrm{d} \boldsymbol{y}}{\mathrm{d} t} \cdot \nabla_{y} \varphi(x, y) \mathrm{d} s \mathrm{~d} t
\end{aligned}
$$


As shown in Equation (8) DeTurck and Gluck extended the linking integral to $S^{3}$ and $H^{3}$, by retaining the $\mathfrak{R}^{3}$ equation and specializing the fundamental solution of the Laplacian in $\mathfrak{R}^{3}$. This clever translation from Euclidean-specific coordinate-based function $r / r^{3} \rightarrow \nabla\left(r^{-1}\right) \rightarrow \varphi\left(r^{-1}\right)$ to generalized Green's function inverse operator vastly extends the topology range of linking.

\section{Derivation of Field Solution from Discrete Inverse Operators}

Recently an alternative technique of inverting field equations developed [8] based on exact discrete inverse operators. For magnetic field Equation (11) describing the circulation of the field induced by current density $\boldsymbol{j}$ we use the discrete inverse curl operator

$$
\nabla \times \boldsymbol{f}=\boldsymbol{j} \Rightarrow \boldsymbol{f}=(\nabla \times)^{-1} \boldsymbol{j},
$$

where $(\nabla \times)^{-1}$ is proved to be $(\boldsymbol{r} \times)$ :

$$
\nabla \times \boldsymbol{f}=\boldsymbol{j} \Rightarrow \boldsymbol{f}=\boldsymbol{r} \times \boldsymbol{j}
$$

In Euclidian 3-space $\mathfrak{R}^{3}$, the classical convolution formula of Biot and Savart gives the magnetic field $\boldsymbol{B}(\boldsymbol{j})$ of a compactly supported current flow $\boldsymbol{j}$ :

$$
\boldsymbol{B}(\boldsymbol{j})(y)=\frac{1}{4 \pi} \int_{\mathfrak{R}^{3}} \mathrm{~d}^{3} \boldsymbol{x} \boldsymbol{j}(\boldsymbol{x}) \times \frac{\boldsymbol{y}-\boldsymbol{x}}{|y-x|^{3}}
$$

Of course Biot and Savart did not derive this equation from a convolution formula; they derived it based on experiments with current carrying wires, compass needles, and torsion balances. Physically, if current flows in a wire loop, the circulation of the resulting magnetic field around a second disjoint from it is equal to the flux of the current through a cross-section of the wire loop multiplied by the linking number of the two loops. An example is shown in Figure 2. This of course agrees with loop-based formulas for induction in transformers and in solenoidal magnets.

Consider Ampere's law of magnetic circulation [9],

$$
\boldsymbol{\nabla} \times \boldsymbol{B} \sim \boldsymbol{J}
$$

based on charge current density $\boldsymbol{J}=\rho \boldsymbol{v}=q \boldsymbol{v} / r^{3} \quad$ [where $\sim$ implies scale factor]. The discrete inverse curl operator $(\nabla \times)^{-1}=(\boldsymbol{r} \times)$ solves for magnetic field $\boldsymbol{B}$

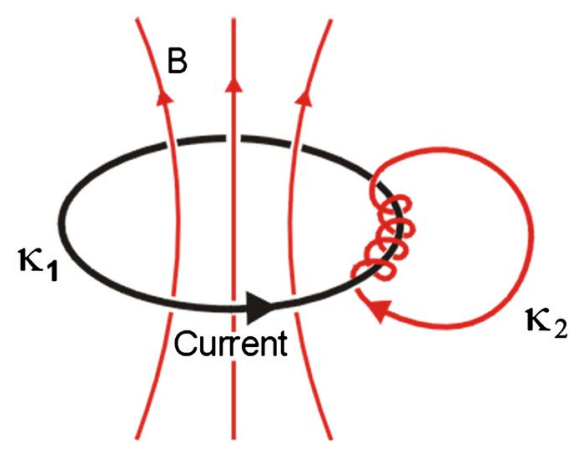

Figure 2. Multi-linking example. 
in terms of current density $\boldsymbol{J}$ :

$$
\boldsymbol{B}=\boldsymbol{r} \times \boldsymbol{J}=\boldsymbol{r} \times \frac{q \boldsymbol{v}}{r^{3}}=\frac{\boldsymbol{r}}{r^{3}} \times q \boldsymbol{v}=\frac{\boldsymbol{r}}{r^{3}} \times \boldsymbol{j}
$$

where we let $\boldsymbol{j}=q \boldsymbol{v}$ represent charge $q$ moving with velocity $\boldsymbol{v}$, such that the magnitude of the magnetic field induced at position $r$ with respect to $q$ is [ignoring signs]:

$$
\boldsymbol{B}(\boldsymbol{r})=\boldsymbol{j} \times \frac{\boldsymbol{r}}{r^{3}}=\boldsymbol{j} \times \nabla\left(\frac{1}{r}\right) .
$$

\section{The Biot-Savart Formula and Helicity}

Based on Figure 1 we identify the tangent to the $\kappa_{1}$-curve as current at $y$. $\boldsymbol{j}=\mathrm{d} y / \mathrm{d} t$ and the tangent to the $\kappa_{2}$-curve as magnetic field $\boldsymbol{B}=\mathrm{d} x / \mathrm{d} s$ and substitute these into Equation (1) to obtain:

$$
\operatorname{Link}\left(\kappa_{1}, \kappa_{2}\right)=\frac{1}{4 \pi} \int_{\kappa_{1} \times \kappa_{2}} \boldsymbol{B} \times \boldsymbol{j} \cdot \frac{\boldsymbol{x}-\boldsymbol{y}}{|\boldsymbol{x}-\boldsymbol{y}|^{3}} \mathrm{~d} s \mathrm{~d} t
$$

We use vector identity $\boldsymbol{A} \cdot(\boldsymbol{B} \times \boldsymbol{C})=\boldsymbol{B} \cdot(\boldsymbol{C} \times \boldsymbol{A})$ to re-arrange $\left(\boldsymbol{r} / r^{3}\right) \cdot(\boldsymbol{B} \times \boldsymbol{j})$ and Equation (16) to interpret the result as follows:

$$
\boldsymbol{B} \cdot\left(\boldsymbol{j} \times \frac{\boldsymbol{r}}{r^{3}}\right) \Rightarrow \boldsymbol{B} \cdot \boldsymbol{B}
$$

In order to further understand the meaning of this term, we discuss the fact that, independently, Calugareanu [10] defined a real-valued invariant of a smooth simple closed curve in $\mathfrak{R}^{3}$ by allowing the two curves in Gauss's linking integral to come together. In the limit, points $x(s)$ and $y(t)$ now run along the same curve in Euclidean 3 -space $\mathfrak{R}^{3}$, with linking number [Equation (1)]

$$
\operatorname{Link}\left(\kappa_{1}, \kappa_{2}\right)=\frac{1}{4 \pi} \int_{\kappa_{1} \times \kappa_{2}} \frac{\mathrm{d} \boldsymbol{x}}{\mathrm{d} s} \times \frac{\mathrm{d} \boldsymbol{y}}{\mathrm{d} t} \cdot \frac{\boldsymbol{x}-\boldsymbol{y}}{|\boldsymbol{x}-\boldsymbol{y}|^{3}} \mathrm{~d} s \mathrm{~d} t
$$

The helicity of a vector field $\boldsymbol{v}(\boldsymbol{x})$ defined on a boundary domain $\Omega$ in $\mathfrak{R}^{3}$ is given by

$$
\operatorname{Heli}(\Omega, \Omega)=\frac{1}{4 \pi} \int_{\Omega \times \Omega} \boldsymbol{v}(\boldsymbol{x}) \times \boldsymbol{v}(\boldsymbol{y}) \cdot \frac{\boldsymbol{x}-\boldsymbol{y}}{|\boldsymbol{x}-\boldsymbol{y}|^{3}} \mathrm{~d}^{3} x \mathrm{~d}^{3} y .
$$

The integral is over the volume elements and vector field $\boldsymbol{v}(\boldsymbol{x})$ is a flow density. Helicity is a measure of the extent to which the orbits of $v$ wrap and coil around each other. The potential problem of $1 /|x-y|$ is compensated by terms in the denominator that approach zero faster than $\varphi(\alpha)$. This new invariant measures the extent to which the curve wraps and coils around itself, and, per Parsley [11]: "the helicity of a vector field is bounded by its $L^{2}$ energy." Energy density of the B-field is $B^{2}$, which corresponds to Calugareanu's construction $\kappa_{1} \times \kappa_{2} \rightarrow \kappa \times \kappa$. Calugareanu's specialization of Gauss-linking to twisting, writhing, and helicity, is considered relevant to self-linking into solitonic structures. The application of these concepts spans the biophysics of DNA 
helices, plasma fields in solar winds, knot theory, etc. The end goal of this work will be the determination of a self-linking, self-curling stable field construction. If such exists, I postulate it will have a boundary threshold, below which stability does not hold.

\section{Link Duality}

Duality is a complicated concept. Electric field $\boldsymbol{E}$ and magnetic field $\boldsymbol{B}$ are dual in that the transformation $\boldsymbol{E} \rightarrow \boldsymbol{B}, \boldsymbol{B} \rightarrow-\boldsymbol{E}$ satisfies Maxwell's equations. In geometric algebra the duality operator $\hat{i}$ transforms elements to their dual, for example $\boldsymbol{a} \wedge \boldsymbol{b}=-i \boldsymbol{a} \times \boldsymbol{b}$ converts the bivector $\boldsymbol{a} \wedge \boldsymbol{b}$ into the (axial) vector cross product $\boldsymbol{a} \times \boldsymbol{b}$. We now consider the interesting duality of $\boldsymbol{B}$ and $\boldsymbol{j}$ implied by the linking number. The Figure 1 topology has dual nature; $\kappa_{1}$ can represent the magnetic field $\boldsymbol{B}(\boldsymbol{x})$ induced by the current density $\boldsymbol{j}(\boldsymbol{y})$ at $\boldsymbol{y}$ on $\kappa_{2}$ curve, but it can also represent the magnetic field at $\boldsymbol{B}(\boldsymbol{y})$ on $\kappa_{2}$ induced by current density $\boldsymbol{j}(\boldsymbol{x})$ on $\kappa_{1}$.

The mathematical form of the linking integral shown in Figure 1 is such that the linking number depends neither on the choice of surface (bounded by curves) nor on which of the curves is used to bound the surface. Interestingly this "equivalence" extends to the case shown in Figure 3, in which the source current $\boldsymbol{J}$ can be assigned to $\kappa_{1}$ with the induced field $\boldsymbol{B}$ represented by $\kappa_{2}$, or these can be reversed and the physics is equivalent. This differs from the principle of covariance. These linkages are shown in Figure 3 and obey the Link duality formula:

$$
\operatorname{Link}\left[\kappa_{1}(\boldsymbol{B}), \kappa_{2}(\boldsymbol{j})\right] \equiv \operatorname{Link}\left[\kappa_{1}(\boldsymbol{j}), \kappa_{2}(\boldsymbol{B})\right]
$$

as seen in Figure 3.

\section{Helical Duality}

We observe that the radius of $\kappa_{2}$ can be expanded without limit so the curve at point $y$ becomes essentially a straight line parallel to the tangent $\mathrm{d} y / \mathrm{d} t$ while curve $\kappa_{1}$ remains unchanged. In fact it is quite fascinating that both physical situations retain meaning in this case and display a duality or symmetry of $\boldsymbol{J}$ and $\boldsymbol{B}$. This interesting extension of link duality exhibits scale invariance of the
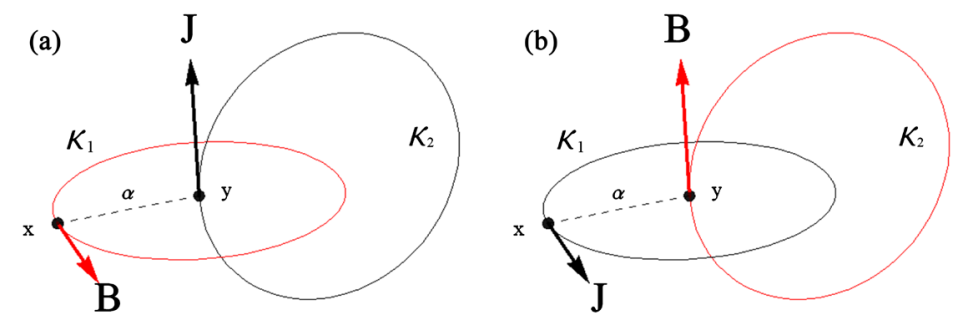

Figure 3. The linking duality is such that the linking number depends neither on the choice of surface nor which of the curves is used to bound the surface. In (a) current $J$ induces field $\boldsymbol{B}$ in $\kappa_{1}$ boundary, while in (b) current in the $\kappa_{1}$ boundary induces field $\boldsymbol{B}$ in $\kappa_{2}$. 
radius of curve when the radius of $\kappa_{2}$ grows without limit and the segment containing point $y$ becomes a straight line parallel to the tangent, $d y / d t$. In Figure 4 we illustrate the duality that is expressed as $(\nabla \times)^{-1} \boldsymbol{J}=\boldsymbol{B} \Leftrightarrow(\nabla \times) \boldsymbol{B}=\boldsymbol{J}$.

Symbolically, the discrete inverse curl operator $(\nabla \times)^{-1} \sim \frac{1}{\left(\frac{\partial}{\partial r} \times\right)} \rightarrow(\boldsymbol{r} \times)$ therefore we focus on the physical meaning of $\boldsymbol{r}$, which is the directed distance from $x$ on the source curve to $y$ on the induced curve. In Figure 4(a), the current source density $\boldsymbol{J}$ is in the wire helix with radius $|\boldsymbol{r}|$ centered on the axis of the helix. We consider the induced $\boldsymbol{B}$ field along the axis of the helix and the field is defined by $\boldsymbol{B}=\boldsymbol{r} \times \boldsymbol{J}$ as indicated. In Figure 4(b) the current density $\boldsymbol{J}$ flows along the center of the helix and the induced $\boldsymbol{B}$ field lies on the helix with radius $|\boldsymbol{r}|$ and is once again defined as $\boldsymbol{B}=\boldsymbol{r} \times \boldsymbol{J}$.

\section{Extending Duality}

Linking and helicity have been defined as integrals and associated with current source density $\boldsymbol{J}$ that induces an electromagnetic field $\boldsymbol{B}$ via the Ampere Law and the Law of Biot-Savart. As noted above, "duality" is an interesting concept. Most examples are essentially "one-dimensional"; the electromagnetic field example $\{\boldsymbol{E}, \boldsymbol{B}\} \Leftrightarrow\{\boldsymbol{B},-\boldsymbol{E}\}$ swaps two fields and changes the sign (direction) of one of the fields. The duality operator in geometric algebra exchanges one geometric algebra entity with its dual entity $\{$ vector $\} \Leftrightarrow\{$ bivector $\}$, etc. What we have revealed above is a more complex duality involving fields, currents, geometry, and topology. We now ask whether this can be pushed further, and we do so using electromagnetic induction as an example.

$$
\boldsymbol{J} \stackrel{?}{=} \boldsymbol{B} \cdot \boldsymbol{B}
$$

The key aspect of the duality discussed above is that the helical current induces a linear field at its center and a linear current induces a helical field surrounding it. The current has charge, mass, and momentum, while the magnetic field has energy density and angular momentum. The helical duality shown in Figure 4 is geometrical and topological. The linking is directly physical, in the sense that current flow through the surface bounded by a curve will induce a field circulation in the boundary tangent to the boundary. The inverse operation is more subtle; if the change in field occurs in a current carrying boundary, an electromotive force (a voltage) is induced that generates a change in current. The formal description of this physics is given by Lenz's Law. Hence there is a certain

(a)

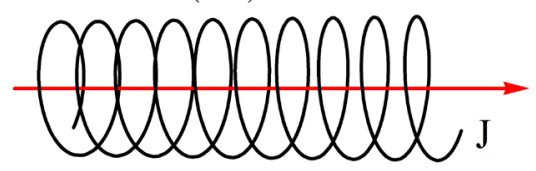

(b)

$\mathrm{B}$

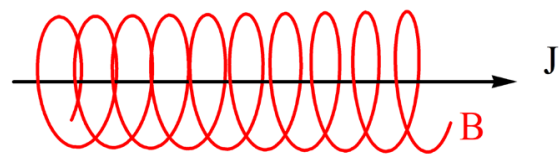

Figure 4. Solenoidal dual helical aspects of electromagnetism. The unique duality allows both source and field to link to helix of other entity. 
symmetry associated with the linking, but there is an indirectness (the "emf") that corresponds to a certain asymmetry as well.

The basic experiments from which the laws were derived utilize metallic wire to conduct the charge current, but the electromagnetic laws operate without wire, as in charged plasma current flows distributed over space.

$$
\left\{\begin{array}{c}
\boldsymbol{J} \\
\text { mass } \\
\text { charge } \\
\text { momentum }
\end{array}\right\} \neq\left\{\begin{array}{c}
\boldsymbol{B} \cdot \boldsymbol{B} \\
\text { mass } \\
\text { uncharged } \\
\text { momentum }
\end{array}\right\}
$$

What we're asking in this section is how one might extend the linking phenomenon such that the field might link to itself. From the relation between the current $\boldsymbol{J}$ that induces the field, and the energy density (with mass-density-equivalence) of field $\boldsymbol{B} \cdot \boldsymbol{B}$ we observe that the field is uncharged and therefore cannot act upon itself in the manner that charge acts to produce the field. Similarly, the field acts upon the charge of the current flow. So it is electric charge that is at the heart of this physical phenomenon and this represents a basic asymmetry since the field is always inherently uncharged.

Our conclusion is that the electromagnetic field of Maxwell's laws will not support the hoped-for extension of duality. There is, however, an alternative, which we look at next.

\section{A Self Linking Field}

The above analysis indicates that the absence of charge of the electromagnetic field prevents the field from linking to itself in a "self-dual" matter. Is any other field "self-dual" as described here?

In 2011 the Gravity Probe B experiment proved the existence of the gravitomagnetic field, first proposed by Oliver Heaviside in 1893. I've recently published papers on different aspects of gravito-magnetism and will not go into much depth in this paper except on the question of self-linking. The gravitational field resolves into a gravito-electric field $\boldsymbol{G}$ analogous to the electric field $\boldsymbol{E}$ and a gravitomagnetic field $\boldsymbol{C}$ analogous to magnetic field $\boldsymbol{B}$. The analogy is mathematical, the gravitational field does not possess electric charge, nor does the gravitomagnetic C-field act on electric charge, per se. The gravito-dynamic equations however are almost identical to Maxwell's equations when electric charge density is replaced by mass density. Here we focus on the C-field analogy with the B-field and observe:

$$
\begin{aligned}
& (\nabla \times)^{-1} \boldsymbol{J}=\boldsymbol{B}, \quad(\nabla \times) \boldsymbol{B}=\boldsymbol{J} \\
& (\nabla \times)^{-1} \boldsymbol{P}=\boldsymbol{C}, \quad(\nabla \times) \boldsymbol{C}=\boldsymbol{P}
\end{aligned}
$$

where scalar constants $(g=c=1)$ have been set to one. From previous sections we conclude that the momentum density $\boldsymbol{P}=\rho_{m} \boldsymbol{v}$ induces a $\boldsymbol{C}$-field circulation around $\boldsymbol{P}$ analogous to the charge density flow $\boldsymbol{J}=\rho_{q} \boldsymbol{v}$ inducing a $\boldsymbol{B}$ 
-field circulation around $\boldsymbol{J}$. We expect the helical topology shown in Figure 4 to describe gravito-magnetism, and it does. However there is an aspect of the $\boldsymbol{C}$-field that is missing from the magnetic $\boldsymbol{B}$-field-the $\boldsymbol{C}$-field interacts with itself! Like the $\boldsymbol{B}$-field, the $\boldsymbol{C}$-field has energy density and momentum density and we see from Equation (23b) that momentum density induces $C$-field circulation. This newly induced $\boldsymbol{C}$-field circulation possesses (mass) energy density and momentum density and hence induces still more $C$-field circulation, etc. as shown in "Iterating with Fuzzy Parameters...". Compare this result with the relation

$$
\left\{\begin{array}{c}
\boldsymbol{P} \\
\text { mass } \\
\text { momentum }
\end{array}\right\} \cong\left\{\begin{array}{c}
\boldsymbol{C} \cdot \boldsymbol{C} \\
\text { mass } \\
\text { momentum }
\end{array}\right\}
$$

We see that the momentum $\boldsymbol{P}$ that induces $\boldsymbol{\nabla} \times \boldsymbol{C}$ produces momentum associated with field energy density $\boldsymbol{C} \cdot \boldsymbol{C}$ and this field thus links to itself, which is what we were searching for.

The physics, geometry, and topology all support self-linking in the dualistic manner described. The nature of the cross product is orthogonality, so that circulation induced by the momentum at distance $\boldsymbol{r}$ from the momentum is orthogonal to the momentum. We next look more closely at the induced field orthogonal to the momentum. Einstein and deHaas [12] experimentally proved that the magnetic field possesses angular momentum. In fact, the gravitomagnetic field is mathematically identical to angular momentum when one ignores the scalar constants.

$$
\begin{aligned}
\boldsymbol{L} & =\boldsymbol{r} \times \boldsymbol{P} \\
\boldsymbol{C} & \sim \boldsymbol{r} \times \boldsymbol{P}
\end{aligned}
$$

where the $\sim$ indicates a scale factor $\left(g / c^{2}\right)$ is required.

In other words the gravitomagnetic $C$-field circulation actually is physically circulating! For instance the Gravity Probe B detection [13] of the "Lenz-Thirring" effect is thought of in metric terms as 'frame dragging', but in actuality, the $\boldsymbol{C}$-field is in motion, and this motion imparts a momentum density to the energy density of the $C$-field.

This momentum density $\boldsymbol{P}^{\prime}$ of the induced field is, as noted, orthogonal to the inducing momentum $\boldsymbol{P}$, and the second order induction arising from $\boldsymbol{P}^{\prime}$ will be orthogonal to $\boldsymbol{P}^{\prime}$. We depict this in Figure 5 where red and green are used to emphasize orthogonality. Red is parallel to red and green is parallel to green, while red and green are orthogonal to each other.

We note explicitly the colors (red and black) in earlier diagrams were used to distinguish between charge current (black) and physical field (red). Our use of color here is to distinguish directions of momentum associated with higher order induction. The key result we wish to focus on here is the fact that while the first-order induction is orthogonal to the inducing momentum, the second-order induction of the induced momentum has a component that is in exactly the 


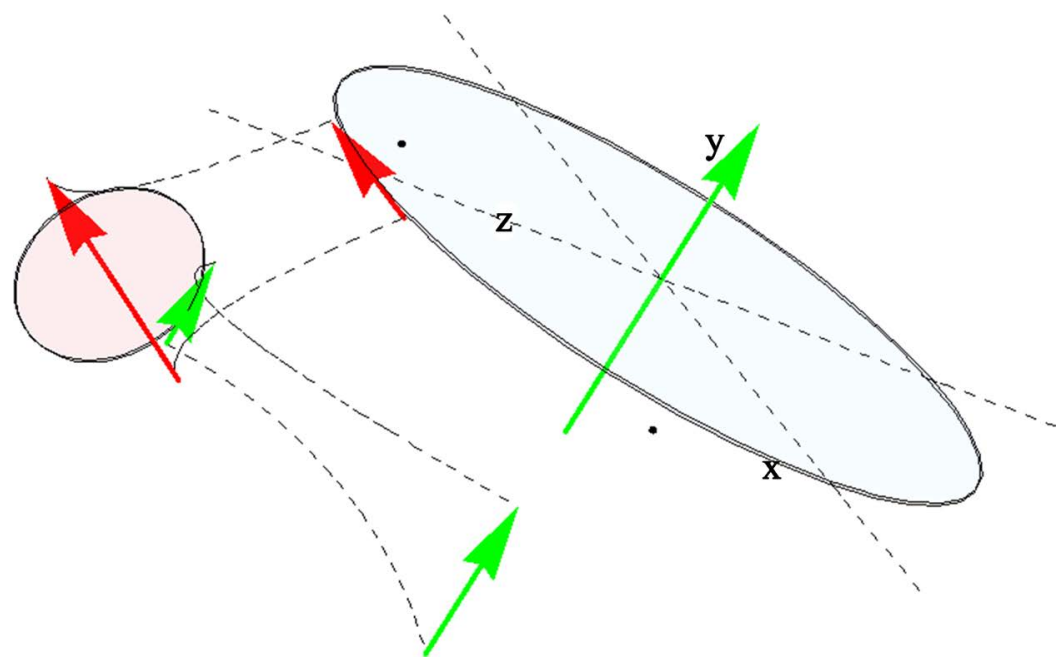

Figure 5. Color-coded diagram of first and second order induction of $C$-field.

same direction as the first-order momentum. This type of feedback suggests stability and thus we have grounds to hope that a self linking field may support stable field configurations.

Two aspects are key: first, the induced $\boldsymbol{C}$-field momentum induces a second order $\boldsymbol{C}$-field circulation. This circulation has one component, shown in Figure 5, that self-reinforces the original momentum, but the same circulation has another component (only implicitly shown) that exactly opposes the original momentum. But the reinforcing momentum component is closer to the original momentum, and thus the reinforcing interaction is stronger than the opposing interaction, as would be required for self-stabilizing field configurations.

Second, the Heaviside gravitomagnetic equations are generally known as the "weak field approximation" and as such are argued not to produce higher order self-interaction. This is addressed in The Primordial Principle of Self-Interaction where it is shown that "weak field" is a misnomer. The Heaviside equation is derived in a strength-independent manner, assumed valid even at the big bang, therefore the equation is for all fields, not just weak fields. Clifford Will and others have remarked on the surprising accuracy of the equation for strong fields.

\section{Conclusion}

We have reviewed topological linking and helicity formulas and discussed the relation of these concepts to electromagnetic phenomena. I have then introduced and focused on the concept of duality in this context and asked whether a self-dual or self-linking field exists which might support self-stabilized field configurations. I then review the nature of the gravitomagnetic field equations in terms of the self-dual linking and helicity that we were searching for. The combination of the mathematics, the physics, the geometry and the topology lends a certain complexity to this enterprise, but I have produced a schematic proof that the self-dual gravitomagnetic field density supports higher order stabilization 
through self-dual self-induction.

To put things in context, Wheeler [14] believed that nature would avail itself of all opportunities offered by the equations of valid theories. In particular he defined a "geon" as a gravitating body made entirely of electromagnetic fields and hoped that they would give "mass without mass", but later showed that these are unstable-they would quickly self destruct if they were ever to form. I have shown herein that, although electromagnetic field structures are unstable, gravitomagnetic fields support self-stabilizing structure that may lead to self-stabilized, soliton-like structures that represent a resonance or even enduring entities. I plan to follow this paper with analysis of such.

\section{Conflicts of Interest}

The author declares no conflicts of interest regarding the publication of this paper.

\section{References}

[1] Klingman, E. (2020) Journal of Modern Physics, 12, 65-81. https://doi.org/10.4236/jmp.2021.122007

[2] Klingman, E. (2019) Applied Mathematics, 11, 1070-1080. https://doi.org/10.4236/am.2020.1111072

[3] Will, C. (2011) PNAS, 108, 5938-5945. https://doi.org/10.1073/pnas.1103127108

[4] Klingman, E. (2019) Prespace-Time Journal, 10, 671-680.

[5] Vishwakarma, R. (2013) Einstein”s Real “Biggest Blunder”. https://fqxi.org/community/forum/topic/1840

[6] DeTurck, D. and Gluck, H. (2008) Journal of Differential Geometry, 94, 87-128. https://doi.org/10.4310/jdg/1361889062

[7] Klingman, E. (2020) Journal of Modern Physics, 11, 1950-1968. https://doi.org/10.4236/jmp.2020.1112123

[8] Klingman, E. (2020) Journal of Applied Mathematics and Physics, 8, 2213-2222. https://doi.org/10.4236/jamp.2020.810166

[9] Jackson, J. (1962) Classical Electrodynamics. John Wiley, New York, 137-138. https://doi.org/10.1063/1.3057859

[10] Calugareanu, G. (1961) Czechoslovak Mathematical Journal, 11, 588-625. https://doi.org/10.21136/CMJ.1961.100486

[11] Parsley, R. (2004) The Biot-Savart Operator Electrodynamics on Bounded Subdomains of the Three-Sphere. PhD Thesis, University of Pennsylvania, Philadelphia.

[12] Einstein, A. and deHaas, W. (1915) Experimental Proof of Existence of Ampere's Molecular Currents. KNAW Proceedings, Amsterdam, 1915, 696-711.

[13] Everitt, C.W.F., et al. (2011) Physical Review Letters, 106, Article ID: 221101.

[14] Wheeler, J. (1998) Geons, Black Holes, and Quantum Foam. WW Norton and Co., New York. 